\title{
KOGNITIVE UND SEMANTISCHE ASPEKTE VON VERWENDUNG DES LEXEMS TON IN DER DEUTSCHEN UND IN DER SPANISCHEN PHRASEOLOGIE ${ }^{1}$
}

\author{
ANA MANSILla PEREZ \\ Universitat de Murcia \\ anamansi@um.es
}

\section{ZUSAMMENFASSUNG}

Das Ziel dieses Beitrags besteht darin, das Verwendungsspektrum der Lexeme dt. Ton und sp. tono (als Bezeichnung für den natürlichen Klang und den Klang in der Sprache) in Phraseologismen des Deutschen und des Spanischen zu ermitteln und dem möglicherweise motivierten Zusammenhang zwischen wörtlicher und figurativer Bedetung auf den Grund zu gehen. Anhand des musikalischen Parameters Ton soll die phraseologische Produktivität der deutschen und der spanischen Sprache vergleichend untersucht und der Frage nachgegangen werden, inwieweit bestimmte Mitteilungen je nach Klangfarbe (Ton/Stimme) bzw. Intonation (Rede- und Sprechweise) metaphorisch relevant sind und ob sie zur Bewertung der Kommunikationssituation beitragen können.

SCHLÜSSELWÖRTER: Phraseologie, Ton, Musik, deutsch, spanisch, Metapher

SEMANTIC AND COGNITIVE ASPECTS OF THE USE OF THE LEXEME TONE IN
GERMAN AND SPANISH PHRASEOLOGY

\section{ABSTRACT}

The purpose of this paper is to analyze the use of the lexemes 'Ton' and 'tono' (concepts relating to both natural sound and sound in language) in German and Spanish phraeologisms and to study the relationship between the literal, figurative and/or motivated meaning of such phraseologisms. Taking the musical parameter 'Ton' as its basis, the paper conducts a contrastive exploration of phraseological production in German and Spanish and seeks to establish, on the one hand, how human communication in terms of tone, voice and intonation may shed light on language's metaphorical dimension and, on the other, whether this can contribute to a clearer assessment of any communicative situation.

KEYWORDS: Phraseology, Ton, Music, German, Spanish, Metaphor

${ }^{1}$ Diese Arbeit ist im Rahmen des Forschungsprojekts Combinaciones fraseológicas del alemán de estructura [Prep. + Sust.]: patrones sintagmáticos, descripción lexicográfica y correspondencias en español (FFI2013-45769-P) entstanden, welches von Frau Dr. Carmen Mellado Blanco geleitet wird. 


\section{EINFÜHRUNG}

Die Musik spielt im Alltag zweifelsohne eine wichtige Rolle. Dies kann erklären, weshalb musikalische und akustische Begriffe wie Schwingung, Klangfarbe, Takt, Ton u.a. in zahllosen festen Worverbindungen auftauchen. Wenn solche Idiome einen lexikalisierten Status erreichen, wird mit Schwingung, Ton, Stimmung in der Regel nicht mehr auf die Musik verwiesen. Ist es kognitiv nachweisbar, warum man den richtigen Ton treffen muss, wenn man über zwischenmenschliche Beziehungen sprechen möchte? Und warum findet ausgerechnet das Wort Ton Verwendung, das in zahlreichen idiomatischen Ausdrücken vorkommt (sich im Ton vergreifen, einen freundlichen Ton anschlagen, der Ton macht die Musik, usw.), welche wenig mit Musik zu tun haben? Kann man überhaupt von Musik als konzeptuelle Metapher sprechen, wenn keine konkrete Quelldomäne systematisch benannt werden kann? Wir gehen von der Prämisse aus, dass der metaphorisch oder metonymisch benutzte Ausdruck Ton eine körpergebundene Quelldomäne besitzt. In Anlehnung an die Metapherntheorie Lakoffs und Johnsons (1980) werde ich versuchen aufzuzeigen, inwiefern metaphorische Modelle einen Erklärungsansatz liefern können.

Für das Spanische und das Deutsche existiert noch keine vergleichbare und umfangreiche Studie, die sich einerseits mit Phraseologismen aus dem musikalischen Bereich befasst und andererseits Betrachtungsweisen und Erkenntnisse der strukturalistischen und der kognitiven Linguistik in ihre Darstellung integriert. Die vorliegende Arbeit setzt es sich zum Ziel, diese Forschungslücke teilweise zu schließen. Das empirische Material, das der vorliegenden Arbeit zu Grunde liegt, ist aus verschiedenen einsprachigen und zweisprachigen phraseologischen Wörterbüchern entnommen.

\section{MUSIK UND KOGNITIVE LINGUISTIK. DER TON AUS KOGNITIVER SICHT}

Es würde den Rahmen dieser Arbeit sprengen, detailliert auf Untersuchungen zur Musik als Sprache oder zur Sprache als Musik einzugehen. Daher sollen lediglich allgemeine Betrachtungsweisen zur Musik innerhalb der metaphorischen Sprache überblicksartig dargestellt werden.

Wenn wir mit Metaphern operieren, projizieren wir bestimmte Typen von mentalen oder kognitiven Modellen aus unserer Erfahrungswelt auf die Musik. Wir können z. B. sagen, dass wir den Ton anheben, oder dass wir einen anderen Ton anschlagen, ganz so als ob wir als Musikinstrumente auftreten würden. Bestimmte musikalische Parameter lassen sich sprachlich veranschaulichen, indem wir sie auf konkrete menschliche Ereignisse projizieren.

Sprache und Musik weisen viele Gemeinsamkeiten auf, aber sie gehören zu unterschiedlichen Systemen. Die Verschiedenartigkeit der Einsatzbereiche von Sprache und Musik hängt damit zusammen, dass die menschliche Sprache vordergründig mit begrifflichem Denken verbunden ist, was bei Musik nicht im 
gleichen Maße der Fall ist (Vieregge 2000: 163). Was die Musik und die Sprache dennoch verbindet, ist die Akustik. Die prosodischen Parameter der Sprache wie die Intonation, die Lautdauer oder die Lautintensität, welche für die Sprachmelodie oder den Sprachrhythmus ausschlaggebend sind, sind der Musik sehr ähnlich. Beispielsweise bezeichnet der Ton innerhalb der Akustik ein Schallereignis mit einer bestimmten Grundfrequenz. Entsprechend ist der musikalische Ton eine periodische Schwingung, welche mit der Tonhöhe der Tondauer, der Lautstärke und der Klangfarbe verglichen werden kann.

Der eigentliche Ursprung eines Tones sind Schallwellen, welche an unser Ohr dringen, an das Gehirn weitergeleitet und dort verarbeitet werden und schließlich Musik in unserer Wahrnehmung erzeugen. Somit kann man behaupten, dass der musikalische Ton eine physikalische Basis besitzt (Akustik), auf welche eine Verarbeitung im Gehirn folgt (Medizin, Neurologie) die schließlich zu einer geistigen Wahrnehmung führt (Psychologie).

Betrachtet man das Lexem Ton, das laut DUDEN über mindestens sieben Sememe (Lesarten) verfügt, so kann festgestellt werden, dass Ton polysem ist:
a. vom Gehör wahrgenommene gleichmäßige Schwingung der Luft, die
b. keine Obertöne aufweist: ein hoher, tiefer Ton
c. aus einer Reihe harmonischer Töne zusammengesetzter Klang: ein klarer, runder Ton, ein halber Ton
d. Tonaufnahme: einen Film mit Ton unterlegen
e. Rede-, Sprech-, Schreibweise: der Ton seines Briefes ist arrogant
f. Wort; Äußerung: keinen Ton reden, verlauten lassen.
g. Betonung, Akzent: die zweite Silbe trägt den Ton.
h. Farbton: warme, matte Töne

Der Ton kann mit verschiedenen Bereichen in Verbindung gebracht werden, wie z.B. mit dem Sprach- und Wortklang, mit der Lautstärke im Film, oder mit der Art und Weise, wie man sich beim Reden oder beim Schreiben verhält.

\section{DER TON/SPRACHKLANG IST EINE [ERTASTBARE / GESCHMACKLICHE] SUBSTANZ}

Sinneseindrücke spielen eine herausragende Rolle bei abstrakten Denkvorgängen, da sensomotorische Erfahrungen das Gerüst des konzeptuellen und metaphorischen Wissens darstellen. Im Bereich des Tastsinns ist die Haut (Goldstein 2002) das größte Sinnesorgan, in welchem mehrere Rezeptoren für Druck, Berührung, Vibration, Kälte oder Wärme lokalisiert werden können.

Was den Ton als Sprachklang (Stimme) betrifft, hat sich herausgestellt, dass mit jeder Art von Material sinnliche Wahrnehmungen verbunden sind, die zum Auslöser für die sprachliche Metaphorik werden. Ein Material kann als warm, rau, kantig, grob, kalt, feucht oder glatt empfunden werden. Diese Aspekte gehen in das konzeptuelle menschliche Gedächtnis ein. In einer Kultur, in der visuelle Erfahrungen im Vordergrund stehen, ist der Tastsinn als Wurzel menschlicher 
Eindrücke oft übersehen worden. Über das Ertasten kann sich der Mensch nicht nur eine Vorstellung von Räumlichkeit und Grenzen verschaffen, sondern auch von abstrakteren Domänen. Sprachlich tritt der Tastsinn zum Beispiel in den folgenden Ausdrücken in Erscheinung: Dt. hartes Jahr, weiche Drogen, glatter Sieg, kaltes Lächeln, schmieriger Ton, gelinde Strafe, usw.; sp. persona dura, drogas blandas, tela lisa, tiempo áspero, una cálida acogida, sonrisa fría, respuesta cortante, pena suave, usw.

In Verbindung mit dem Lexem Ton werden eine Reihe von Attributen verwendet, welche eng mit dem Tastsinn verbunden sind. So wird z.B. bei einer Sprechweise bzw. bei einer Verhaltensweise von einen harten Ton anschlagen, in einem groben Ton sprechen, einen rauen Ton pflegen gesprochen. Dabei werden die Materialeigenschaften zur Beschreibung von menschlichen Handlungen genutzt. Des Weiteren werden harte, raue und warme Gegenstände durch Emotionalität gekennzeichnet. Hinsichtlich des Materials (hart-weich, warm-kalt; duro-blando; caliente-frío) drückt der Phraseologismus einen harten Ton anschlagen je nach Kontext Strenge, Intensität oder Derbheit aus:

(1) Aber Obamas neue Israel-Politik nimmt Formen an. Er fordert einen echten Siedlungsstopp im Westjordanland. Das haben seine Vorgänger von Ronald Reagan bis George W. Bush auch getan. Aber der Demokrat schlägt einen bisher unbekannt harten Ton an. Auch seine Außenministerin Hillary Clinton verlangte einen bedingungslosen Siedlungsstopp, und zwar "nicht nur für einzelne Siedlungen oder Außenposten, sondern das Ende des natürlichen Wachstums” - ohne Ausnahme. „Israelische Regierungsbeamte sind erschrocken über die harte Linie", notiert die New York Times. (NUN09/MAI.03084 Nürnberger Nachrichten, 30.05.2009, S. 5; Obama bricht ein Tabu -US-Präsident will Israel ernsthaft unter Druck setzen)

(2) Los candidatos a la Junta Susana Díaz (PSOE), Juan Manuel Moreno (PP) y Antonio Maíllo (IU) afrontaron el segundo y último debate a tres, en TVE, en un tono más duro. (El correo de Andalucía 15.03.2015)

Auf der anderen Seite lässt sich der weiche Ton seltener metaphorisch verwenden. Er deutet dabei auf die Spielweise in der Musik (mit einem weichen Ton spielen, blasen, zaubern usw.) oder auf die Farbe hin. Die Suche im DeReKo (das Deutsche Referenz Korpus) ergab in der Kombination weicher Ton wenige Belege, die metaphorisch verwendet werden:

(3) Präsident George Bush und seine Wahlkampfstrategen, die den Texaner in den vergangenen Wochen massiv attackierten, während sich Clinton und die Demokraten hier weitgehend heraushielten, schlagen jedenfalls plötzlich einen weichen Ton an, wenn sie von Ross Perot und seinen Anhängern sprechen. "Das Wichtige ist meiner Meinung nach, was wir von Perot und seinen Anhängern halten", dozierte etwa Bush-QuayleKampagnemanager Fredric V. Malek am Sonntag: "Wir heißen seine Anhänger willkommen." Noch vor kurzem war Perot jener Mann, der, wie Vizepräsident Dan Quayle nicht oft genug darauf verweisen konnte, "eine Geringschätzung für die Verfassung" an den Tag legte. (P92/JUL.21454 Die Presse, 21.07.1992; Ross Perot bleibt auf den Wahllisten) 
Im Spanischen kann der tono duro metaphorisch mit verbaler Aggresivität assoziiert werden. Hingegen ist der tono blando in den untersuchten Korpora kaum belegt:

(4) Y es que la correcta filosofía de este tipo de humor debe basarse en la máxima agresividad, clave del éxito de los muñecos ingleses. El tono blando, ligero, controlado, de los diálogos de sus protagonistas los acerca a las marionetas, y ahí muere su fuerza innovadora. Un muñeco "con vida propia" diciendo lo que jamás se atrevería a decir su homónimo, causaría conmoción pero sería revolucionario. Todo el lenguaje popular, la comidilla de calle, el comentario solapado, el rumor hiriente del ciudadano de a pie puesto en boca de los protagonistas de látex, rompería los índices de audiencia de cualquier televisión. ¿Se imaginan a Juan Guerra hablando al Parlamento? ¿A Aznar debatiendo sobre el caso Filesa? ¿Cuánto hubiera dado de sí el problema de las autonomías? ¿Y el conflicto del teléfono de Benegas? (CREA Apaga y vámonos. La televisión: Guía de supervivencia. Joaquín Carbonell, 1992, S. 203)

In Syntagmen wie harter Ton oder weicher Ton wird die Bedeutung von hartweich durch eine Art Interferenz erweitert, denn diese Verwendungsweise geht über physikalische Phänomene (Tastsinn) hinaus. In diesem Sinne werden Attribute wie weich und hart dem psychologischen Bereich der Handlungsweise bzw. der Verhaltensweise zugesprochen. Der Ausdruck einen harten Ton anschlagen (= streng sein) interagiert mit drei umfangreichen Domänen: mit der physischen Sinneswahrnehmung (harter Gegenstand), dem Bereich psychischen Erlebens und der Welt der sozialen Beziehungen bzw. des zwischenmenschlichen Handelns.

Hinsichtlich der Oberfläche (rau-glatt; rugoso-liso) finden sich die Attribute rau, barsch oder grob. Bei rauem Ton ergaben sich bei der Suche folgende Muster:

in rauem Ton VERBsprechaktverben (diskutieren/sagen/antworten/kommunizieren)

einen rauen Ton VERBinchoative Verben (anschlagen/annehmen)

in rauem Ton VERB musikalische Verben (spielen/blasen)

Durch einen rauen Ton verliert die Kommunikation an Qualität:

(5) Der Bergfex und die deutschen Gipfelstürmer kommunizieren seit Jahren in rauem Ton. Als sich die Spitze des DAV von Messner jetzt wegen ihres Führungsstils in die Nähe der Nationalsozialisten gerückt fühlte, kam es zum Bruch. Der Ungeliebte findet dafür kaum Worte. Messner: "Ich sehe keine Notwendigkeit, das zu kommentieren." (FOC09/MAI.00263 FOCUS, 18.05.2009, S. 51-51; Gipfelstürmer ohne Gefolge)

Dem Beispiel (5) ist zu entnehmen, dass bestimmte Ereignisse dazu führen können, dass sich zwischenmenschliche Verhältnisse verschlechtern, so dass kein normaler Umgangston mehr möglich ist. Der raue Ton wird ebenso verwendet, wenn man sich grob oder unangemessen ausdrückt bzw. verhält:

(6) Ich weiß von vielen, die vom rauen Ton und der unverblümten Rede bei den AAs und den NAs geschockt waren. Da wird nicht lang um den heißen Brei geredet. (WDD11/A01.98771: Diskussion: Anonyme Alkoholiker/Archiv 2007, In: Wikipedia, 
URL:http://de.wikipedia.org/wiki/Diskussion:Anonyme_Alkoholiker/Archiv_2007:

Wikipedia, 2011)

(7) Fahrgäste beklagen sich über den rauen Ton und miesen Service bei den Verkehrsbetrieben: Da wurde ihnen auch schon mal die Türe vor der Nase zugezogen. So geschehen jüngst an der Linie 52 [...](RHZ00/JUN.10267 Rhein-Zeitung, 16.06.2000; Rauer Ton bei den Fahrern)

Die Adjektive barsch und grob werden in gleicher Weise verwendet. Das Adjektiv rau kommt in Verbindung mit Substantiven wie Holz (raues Holz = uneben), Wetter (raues Wetter $=$ unangenehm, kalt), Stimme (raue Stimme $=$ heiser), u.a. vor. Die Domäne des Tastsinns (bzw. der haptischen Wahrnehmung) wird auf eine andere Perzeption nämlich das Hörbare übertragen (Ton=Stimme). Aus diesem Grund scheint es plausibel zu behaupten, dass der raue Ton eine synästhetische Metapher darstellt, wenn man sich auf die Eigenschaften einer Stimme bezieht. Es sind zwei perzeptive Domänen (die taktile und die akustischhörbare), die miteinander wechselwirken (Barcelona 2003). Darüber hinaus deutet der raue Ton in Kombination mit Sprechaktverben wie sagen, antworten, kommentieren, sprechen u.a. auf nicht perzeptive bzw. auf abstraktere Domänen wie zwischenmenschliche Verhältnisse und Verhaltensweisen hin. In diesem Fall liegt keine genuine Form von Synästhesie vor. Nach Richard E. Cytowic (1989: 1) ist Synästhesie "an involuntary joining in which the real information of one sense is accompanied by a perception in another sense".

Im Spanischen wird in Verbindung mit dem Konzept der Oberfläche (glattrau) un tono rugoso, áspero oder rudo verwendet. Die Untersuchung des Korpus CREA (Corpus de Referencia del Español Actual) hat ergeben, dass der tono rudo kaum belegt ist, hingegen wird rudo (=rau) mit Substantiven wie trato, mundo, golpe, hombre, lenguaje oder contrincante in Verbindung gebracht. In Bezug auf das Adjektiv áspero sind die Substantive mundo/suelo/sonido/cerro/genio/debate/rostro oder sistema repräsentativ. Rugoso ist auf taktile Wahrnehmungen beschränkt (rostro/papel/terreno + rugoso). Die Google-Suche hat ergeben, dass der tono rudo häufiger als der tono áspero oder rugoso vorkommt:

(8) El tono áspero de Mariano Rajoy en sus respuestas a los demás portavoces, especialmente, a Pedro Sánchez, ha eclipsado el fondo del debate del estado de la nación [...]. (El País 25.02.15)

Autoren wie Ullmann (1964) und Day (1997) haben festgestellt, dass bei den Sinneswahrnehmungen die größte Empfängerdomäne der akustische Bereich (höherer Sinn) ist, wohingegen der taktile Bereich die wichtigste Spenderdomäne (niederer Sinn) darstellt:

QUELLE $\rightarrow$ taktil ZIEL $\rightarrow$ akustisch

Vogt (2013: 22) geht in ihrem Aufsatz von der Annahme aus, dass die Sprache keine angeborene Fähigkeit sei. Dieser Gedanke liefert eine Erklärung 
dafür, warum der Mensch mit symbolischen bzw. metaphorischen Systemen operiert, wenn es darum geht, die Realität fassbar und begreiflich zu machen. Der Mensch drückt sich mithilfe von Bildern bzw. Metaphern aus, und hiermit sind Analogieschlüsse gemeint (Ähnlichkeitsrelationen zwischen Konzept A und Konzept B). Sie nennt das Beispiel Der Mann ist ein glatter Typ und stützt ihre These auf die Kinder, die in der frühen Phase des Lebens noch nicht imstande sind, aus dieser sprachlichen Kombination psychologische Zusammenhänge zu bilden. Ferner nimmt die Autorin Bezug auf die Frames-Theorie nach Fillmore (1982), die besagt, dass ein Frame verstehensrelevantes Wissen in Rahmen organisiert, die bestimmte Leerstellen enthalten. Jedes Wort evoziert einen Frame, der bestimmte konzeptuelle Wissenseinheiten enthält und sprachliche Ausdrücke beim Sprachverstehen evoziert.

Vogt untersucht zwei synästhetische Metaphern - schreiende Farbe und kalte Wut - , und nimmt dabei Bezug auf das Phänomen der Synästhesie. Insbesondere geht sie der synästhetischen Metaphorik auf den Grund, welche die Produktivität von Perzeption in der konzeptuellen Sprache aufzeigt. Sie unterscheidet zwischen Pseudosynästhesien (verbale perzeptiv-inferentielle Metaphern und abstrakte konzeptuelle Wortverbindungen wie warmes Rot oder scharfe Kritik), Synästhesien in ihren nicht genuinen Formen und Synästhesien in ihren genuinen Formen. In ihrer Arbeit beschreibt sie die synästhetischen Metaphern in ihrer verbalen Kombinatorik von Bezeichnungen aus verschiedenen Perzeptionsbereichen (Vogt 2013: 30). Diese Betrachtungen deuten darauf hin, wie willkürlich manchmal die Sprache gebraucht wird, wenn es um Assoziationen oder kollokative Zusammenhänge geht (kreative Sprache). Beispielsweise spricht sie von graziöser oder glamouröser Stimme, Verknüpfungen, die über die usuellen synästhetischen Kombinationen hinausgehen.

In Bezug auf den Frame STIMME zeigt sich die 'geringe Intensität' u.a. bei den Adjektiven gelinde, weich, zart, hold, fragil oder zierlich, wobei diese Eigenschaften mehreren Domänen entstammen (taktil, visuell). Vogt (2003: 32) führt 11 konstitutive Deskriptoren (Attribute) für den Frame STIMME auf. Hier werden die für die vorliegende Untersuchung wichtigsten Deskriptoren aufgeführt: Tonhöhe (helle Stimme), hohe Intensität (schreiende Stimme), geringe Intensität (sanfte Stimme), Klangfarbe (runde Stimme), Stimmcharakteristika (gebrochene Stimme), Verlaufsformen mit Aspektcharakter (stockende Stimme), Stimme als Träger von Emotionen (traurige Stimme), Stimme als Ausdruck von Verhalten (aggressive Stimme), Merkmale des Stimmträgers (freundliche Stimme), u.a.

Hinsichtlich der taktilen Wahrnehmung (thermische Reize) von warm und kalt in Kombination mit Ton aktiviert unser idealisiertes kognitives Modell der Erfahrung Eigenschaften wie 'das Mitgefühl' oder 'das Gemütliche' bei einem warmen Ton, während der kalte Ton eine Emotionslosigkeit darstellt, da damit eine kontrollierte Rede beschrieben wird. Die Belege aus DeReKo (das Deutsche Referenz Korpus) zeigen, dass die Wortverbindung warmer Ton nur in wenigen 
Fällen als metaphorisch aufzufassen ist, in vielen anderen verwendet der Sprecher den warmen Ton, um auf die musikalische (klangliche) Bedeutung hinzuweisen:

mit warmem Ton VERBmusikalische Verben (spielen/singen/blasen/streichen)

(9) Von Joseph Haydn war dann das Cello-Konzert in C-Dur zu hören. Angela Schwartz spielte es nicht nur mit beseeltem, warmem Ton, sondern konnte dabei auch ihre ganze Virtuosität entfalten. Das Kammerorchester Flawil war ihr dabei ein zuverlässiger und zurückhaltender Begleiter. (A97/JUN.09983 St. Galler Tagblatt, 18.06.1997, Ressort: TB-GO (Abk.); Kammerorchester musizierte leicht und beschwingt)

Der warme Ton ist stark an die Bedeutung 'Nähe' gebunden, und unterliegt den Metaphern FREUNDLICHE REDE IST WÄRME bzw. UNFREUNDLICHE REDE IST KÄLTE und EMOTIONALITÄT IST WÄRME bzw. MANGEL AN EMOTION IST KÄLTE (Baldauf 1997: 106). Baldauf (1997: 97) verweist auf die Konnotationen von heiß/kalt, denen die Kategorie Attributsmetapher zugesprochen wird, folgendermaßen:

Wärme [weckt] in der Regel angenehme Konnotationen, Kälte dagegen [wird] als unangenehm empfunden. Steigt die Temperatur jedoch über eine gewisse Grenze, so kehrt sich das Bild um: Hitze wird zur Bedrohung und Abkühlung wird gesucht (Baldauf 1997: 102)

Die Konzepte HART / SCHWER, DURO / BLANDO und WARM / KALT, CALIENTE / FRIO bilden eine polare Opposition. Zugleich zählen sie zum Erfahrungsschatz aller Menschen. Außerdem enthalten sie in beiden Sprachen identische kognitive Strukturen:

(10) Keinen Moment etwa hatte Henri Desramault (88) von seinem Rollstuhl aus den Mörder seiner Tochter Jeanne-Marie aus den Augen gelassen. Schwer war Fournirets kalter Zynismus, den er im Saal zur Schau gestellt hatte, zu ertragen gewesen. „Wie ein Wilderer“ sei er zur Jagd auf Jungfrauen, seine Obsession, aufgebrochen, ohne zu wissen, ob er „einen Fasan oder ein Kaninchen erbeuten" werde, hatte er in kaltem Ton berichtet. (NUN08/MAI.02978 Nürnberger Nachrichten, 29.05.2008, S. 30; Höchststrafe für das \&\#8222; Monster der Ardennen\&\#8220; Französischer Serienkiller Michel Fourniret nimmt Urteil ungerührt hin \&\#8212; Mehrere Mordfälle bleiben ungeklärt)

(11) „Ist das etwa die Kette, die deine liebe Muttergotthabsieselig bei dem Wohltätigkeitsdinner getragen hat, bei dem wir uns kennen gelernt haben?, fragte Vanessa. "Das ist sie“, sagte Victor in warmem Ton und schenkte seiner Gattin ein nicht weniger strahlendes Lächeln. „Für die beste Frau der Welt. Meine Frau.“ "Oh mein Victor", hauchte Vanessa. "Aber sie glänzte gar nicht so wunderschön damals." „Hab" ich in Paris aufarbeiten lassen“, sagte er verhalten. (HAZ07/DEZ.05797 Hannoversche Allgemeine, 22.12.2007, S. 6; Der Schlips)

Im Spanischen weist beispielsweise das Syntagma tono frío im Nachfeld Partner wie distante/aséptico/cortés/profesional auf. Die Kotextpartner deuten an, dass der tono frío sich als unfreundlich, distanziert und gefühllos erweist. 
Hingegen werden bei dem tono cálido folgende Partner benutzt: cordial/intimista/tranquilizador/condescendiente. Genauso wie im Deutschen wird der tono cálido mit Gefühl und Herzlichkeit assoziiert.

(12) Estáte quieta, Lulú, no te va a servir de nada, en serio... Lo único que vas a conseguir, si sigues haciendo el imbécil, es llevarte un par de hostias - no estaba enfadado conmigo, me hablaba en un tono cálido, tranquilizador incluso, a pesar de sus amenazas-, pórtate bien, no va a ser más que un momento, y tampoco es para tanto [...] (CREA- Almudena Grandes, Las edades de Lulú, 1989, Editores Tusquets (Barcelona), S. 158)

Kälte und Härte gehen im Deutschen Hand in Hand, wie im folgenden Beispiel veranschaulicht wird:

(13) Die Partei diktiert nach Gutdünken seit dem Jahr 2001 als Mehrheitsbeschafferin für die Regierung von Ministerpräsident Anders Fogh Rasmussen Dänemarks extrem scharfe Ausländerpolitik und einen harten, kalten Ton im Umgang mit zugewanderten Muslimen [...] (NKU08/JUN.00482 Nordkurier, 03.06.2008; Racheakt für Karikaturen)

Der Tastsinn kann in Hinblick auf die Oberfläche eines Materials ebenso besagen, ob ein Werkzeug scharf, stumpf oder spitz ist, wie der Phraseologismus einen scharfen Ton anschlagen zeigt. Der scharfe Ton steht zur 'Unhöflichkeit' in enger Verbindung und ist in die metaphorischen Modelle VERBALE AGGRESSION IST PHYSISCHE AGRESSION und VERBALAKTION IST KÖRPERAKTION eingebettet. Ebenso ist er in die kognitive Metapher TÖNE SIND GEFÄHRLICHE OBJEKTE eingefügt (in scharfem Ton sprechen). Das Attribut 'scharf' ist nicht nur zur Beschreibung von Formen gebräuchlich, sondern auch von Geschmack. Die inheränte Semantik des Adjektivs scharf zeigt in den untersuchten Korpora, dass der scharfe Ton in Verbindung mit Verben eingesetzt wird, welche sprachliche Handlungen bezeichnen (Verba dicendi) oder Verben der verbalen Aggresion darstellen.

In scharfem Ton VERB Verba dicendi (sagen/nennen/erklären/sprechen)

In scharfem Ton VERBverba der verbalen Aggresion (kritisieren/angreifen/vorwerfen/rügen)

Der scharfe Ton lässt sich durch das folgende Beispiel belegen:

(14) Wenn du mit diesem Jungen nicht in scharfem Ton sprichst, nimmt der gar nicht ernst, was du sagst, und macht weiter, was er will! (Schemann et al. 2013, 987)

Im Spanischen finden sich folgende Entsprechungen zum Lexem scharf: punzante (spitz), afilado (scharf) und cortante (schneidend). In Verbindung mit dem Lexem tono wird das Adjektiv cortante bevorzugt (en un tono cortante). Dennoch ist scharf lafilado auch häufig in Verbindung mit Zunge bzw. lengua belegt, was sich auf die Metapher WÖRTE SIND WAFFEN zurückführen lässt (Mansilla Pérez \& Mellado Blanco: Im Druck):

Dt. seine Zunge (an jmdm./an einer Sache) wetzen, eine scharfe Zunge haben, eine spitze Zunge haben, mit dem Säbel gegen jmdn. fechten, gegen jmdn. vergiftete Pfeile schießen; Sp. lengua afilada, darse un filo a la lengua, lengua de doble filo, lengua de hacha, lanzar puyas contra alg. usw. 
Aufschlussreich ist zudem die Versprachlichung von SPRACHKLANG (TON) mithilfe des Geschmackssinns, indem das Akustische über das Gustative verbalisiert wird: dt. ein süßer Ton, bittersüßer Ton, herber Ton, saurer Ton; sp. tono agrio, ácido, dulciamargo, dulce. Die gustatorische Wahrnehmung entsteht dadurch, dass auf der Zunge spezifische Bereiche differenziert werden können, deren Rezeptoren entsprechende Empfindungen erzeugen (Gegenfurtner 2003: 47-48).

Die Analyse der Belege hat gezeigt, dass das Attribut süß sowohl im Spanischen als auch im Deutschen als positiv empfunden wird. Dennoch wird im Deutschen der süße Ton häufig mit dem musikalischen Spielen verbunden (mit süßem Ton spielen/erklingen). Demgegenüber deutet das Spanische auf die Redeweise hin: hablar/decir en un tono dulce. Der herbe Ton ist geschmacklich in der Nähe von bitter und ist metaphorisch gleichbedeutend mit harter Ton (unhöfliche Rede): einen herben/harten Ton anschlagen. In ähnlicher Weise wird im Spanischen tono agrio verwendet, um auf eine lieblose Rede hinzuweisen, die Ärger und Unbehagen ausdrückt.

(15) En el mismo tono agrio y severo, José Amedio manifestaba considerarse un «preso político», y decía tajantemente. (El Mundo 01/03/1994)

Sabine Gross (2002: 63) hat einige interessante Überlegungen zu den Äquivalenzen zwischen musikalischen Parametern und perzeptiven Wahrnehmungen einerseits und zwischen musikalischen Parametern und anderen Bereichen andererseits angestellt. Sie kommt zu der Erkenntnis, dass z.B. Tonhöhe mit räumlicher Höhe, mit Helligkeit oder mit Größe in Verbindung gebracht wird. Diese Feststellung erlaubt es, die Schlussfolgerung zu ziehen, dass die Grenzen zwischen den unterschiedlichen Sinneswahrnehmungen durchlässig sind.

Sowohl im Deutschen als auch im Spanischen machen die Kombination mehrerer Geschmacksrichtungen ,bittersüß', ,süßsauer', dulciamargo, agridulce metaphorisch deutlich, dass bestimmte Ereignisse gemischte Eindrücke hervorrufen können, also insbesondere positive und negative zugleich. Die Suche in DeReKo ergab bei dem Syntagma süßsaurem Ton nur einen Treffer und im spanischen Korpus CREA finden sich nur vier Treffer in Zusammenhang mit tono agridulce (ernste und ironische Redeweise).

(16) Doha - Einer ist unpünktlich am Sonntagmorgen. Es handelt sich, das ist gut zu erkennen, um einen namhaften Fußballprofi aus Holland. Der Bursche habe wohl Probleme mit der Weckfunktion seines iPhones und deshalb den gemeinsamen Treffpunkt an der Säbener Straße verpasst, witzelt Louis van Gaal mit bittersüßem Ton. [...] (U11/JAN.00052 Süddeutsche Zeitung, 03.01.2011, S. 29; Nachdenklich auf Reisen) 


\section{DeR TON (SPRACHKLANG) IST VERTIKALITÄT. DER TON IST OBEN/UNTEN}

Die Tonhöhe sowie die Lautstärke lassen sich in fast allen Sprachen der Welt mit den Bezeichnungen für 'hoch' und 'tief' versprachlichen. Im musikalischen Bereich beschreibt die Tonhöhe die Stufe des Tons innerhalb einer Oktave (Rieländer 1982) und stellt damit einen Bezug zur Frequenz musikalischer Töne her. Die eindeutige Bestimmung der Höhe eines Tones umfasst den Frequenzbereich zwischen ca. $30 \mathrm{~Hz}$ bis max. 15.000 Hz. (Grutschus 2009: 134). Außerhalb dieses Bereiches nimmt der Mensch nur Geräusche wahr.

Die Tonhöhe einer Stimme trägt maßgeblich zur Wirkung gesprochener Sprache bei. Der 'Raum', in dem sich der musikalische Ton bewegt, erweist sich als wichtiger Teil unserer Wahrnehmung, da er als Quelle für Metapher fungiert, um Dinge, Ereignisse und Sachverhalte darzustellen. Beispielsweise werden auch Kategorienkonzepte wie EMOTION, MACHT, SOZIALE STELLUNG, GESUNDHEIT, LEBEN, usw. über Räumlichkeit konzeptualisiert (Baldauf 1997: 154). In diesem Sinne können sich komplexe oder abstrakte Konzepte 'im Raum' oder 'außerhalb des Raumes' befinden. Ferner können andere räumliche Schemata unterschieden werden: OBEN vs. UNTEN, TEIL-GANZES oder DRAUßEN-DRINNEN.

Ein in der kognitiven Metapherntheorie oft angeführtes Beispiel im Bereich der Musiktheorie ist die konzeptuelle Metapher HIGH PITCH IS UP/LOW PITCH IS DOWN (Zbikowski, 1997), die beweist, dass die Konzeptualisierung von Ton Bewegungen eines Körpers (nach oben/nach unten) bezeichnet (Zbikowski 2000: 6).

Als besonders aufschlussreich erweist sich in diesem Zusammenhang der kontrastive Vergleich der idiomatischen Wendungen subir el tono und den Ton anheben. Im Deutschen ist jedoch das Idiom seine Stimme erheben häufiger vertreten. Anhand der Verben subir-erheben wird das Bild von 'Bewegung nach oben' konzeptualisiert. Die Stimme wird als ein Objekt dargestellt, das sich in die Höhe bewegt.

Je nach Kontext kann seine Stimme erheben die metaphorische Bedeutung von 'gegen etw. protestieren' oder 'sich für/gegen etwas aussprechen' ergeben. Ein ähnliches Beispiel für das Konzept OBEN ist die Stimme heben (elevar/levantar la voz), das meistens die wörtliche Bedeutung aktiviert:

(17) Dresden, 12. Januar - Es ist nicht leicht, Uwe Backes zu folgen. Der stellvertretende Direktor des Dresdner Hannah-Arendt-Instituts für Totalitarismusforschung spricht langsam und gleich bleibend monoton. Er lässt lange Pausen, in denen seine Pupillen ein wenig hektisch die Bücherregale in seinem kleinen Büro absuchen. Vor allem aber redet der Politikwissenschaftler gleich bleibend leise, ohne nur einmal die Stimme zu heben. [...](U00/JAN.02047 Süddeutsche Zeitung, 13.01.2000, S. 3; In aller Stille ein Aufruhr)

In einigen Zusammenhängen kann die Stimme heben die metaphorische Bedeutung von 'sich über etwas ärgern, zornig werden' enthalten. Folgendes Beispiel soll dies verdeutlichen: 
(18) „Es gibt einen Augenblick der Wahrheit, wenn Mühl die Zwischenrufer zum Schweigen bringt. Er sagt bloß, ohne die Stimme zu heben: "Ich finde das sehr interessant. Noch etwas? Ruhe jetzt!" Mit ebensolchem Zynismus hatte sich der Prophet des Libertären zum Sektenführer aufgeschwungen.“ [...](P98/FEB.06501 Die Presse, 14.02.1998, Ressort: Kultur; Presse-Stimmen)

Der spanische Ausdruck levantar la voz [die Stimme erheben], der mit dem Phraseologismus subir el tono bedeutungsgleich ist, kennt nicht nur eine wörtliche, sondern auch eine metaphorische Bedeutung, und zwar in dem Sinne von 'jdn. anschreien' oder 'jdn. unfreundlich zurechtweisen'. Im Sprachgebrauch wird levantar la voz häufig im negierten Imperativ (¡no me levantes la voz!) verwendet. Dieser Ausdruck lässt das Verhalten eines Kindes seinen Eltern gegenüber als tadelnswert erscheinen. Im Deutschen findet man den Phraseologismus nicht in diesem Ton (mit jmdm.) sprechen.

(19) Gestern muckte wenigstens mal einer auf (Alonso) und spielte seinem Banknachbarn bei McLaren-Mercedes (Hamilton) einen bösen Streich am Ende der Qualifikation (siehe Kasten links). Klar, dass sich Hamilton beim Lehrer mit Schimpfwörtern beschwerte („Macht das nie mehr mit mir"). Worauf der erboste Lehrer sagte: „Und du sprichst nie mehr in diesem Ton mit mir!" (SBL07/AUG.00083 Sonntagsblick, 05.08.2007, S. s18; Zwei durchgeknallte Lehrer und eine grosse Frage:)

Darüber hinaus kann levantar la voz ebenso im Sinne von 'protestieren gegen etw. /jmdn.' und 'sich äußern' benutzt werden:

(20) En este sentido, alentó a los científicos «a levantar la voz para evitar que se produzcan daños irreversibles en nuestra ecología.» (El País 01/10/98)

Die Intonation und die Tonhöhe spielen eine wesentliche Rolle in der menschlichen Kommunikation. Laut Möbius (1993: 8) umfasst der Oberbegriff 'Suprasegmentalia' die Bereiche Intonation, Lautstärke und Quantität, deren akustische Korrelate Sprachgrundfrequenz, Intensität und Dauer am Sprachsignal abgelesen werden können. Die Intonation bezeichnet alle relevanten Bereiche des akustischen Parameters Grundfrequenz auf Silben-, Wort- und Äußerungsebene. Demgegenüber beschreibt das Merkmal Lautstärke alle linguistischen Funktionen des Parameters Intensität. Der Parameter Qualität schließlich bezieht sich auf die Dauer des Sprachsignals.

In der Alltagskommunikation sind bestimmte Konventionen oder Regeln einzuhalten, welche das Zusammenleben und den Umgang mit anderen vereinfachen. Die Lautstärke ist sie mit dem Konzept VERTIKALITÄT eng verwandt. Dies ist für die Bewertung dessen relevant, wie (un)höflich eine Aussage ist. Beispielsweise kann lautes Sprechen u.a. mit Unhöflichkeit, Unkultiviertheit oder Aufdringlichkeit assoziiert werden. Mit anderen Worten lassen sich höfliche Manieren mit suprasegmentalen Elementen wie Lautstärke, rhythmische Gestaltung oder Intonation verbinden. Je nachdem ob eine Äußerung laut, leise, betont oder unbetont ausgesprochen wird, kann sie 
aggressiv, unfreundlich oder hart wirken (Zarend 2015: 104). Wenn man einen guten Eindruck bei jemandem hinterlassen möchte, empfiehlt es sich, die Tonhöhe nicht zu steigern. Generell wird im europäischen Raum mit kräftiger, lockerer und normal tiefer Stimme gesprochen.

Laut Ehrhardt/Neuland (2009: 21) können die Begriffe 'höflich' und 'Höflichkeit' in zwei unterschiedlichen Bedeutungen verwendet werden: im Bereich der menschlichen Charaktereingenschaft und innerhalb des sprachlichen Verhaltens.

Folgende Phraseologismen sind negativ konnotiert und betonen das Konzept $\mathrm{HOCH}$ innerhalb der Sprechweise. Aspekte der Tonhöhe werden metaphorisch auf die Sprechweise bzw. auf die Charaktereigenschaften einer Person übertragen.

Dt. in überheblichem Ton reden, hochtönend reden, einen überheblichen Ton anschlagen, die Stimme erheben; sp. subir el tono, subir de tono, alzar el tono de voz, levantar la voz, hablar en un tono altivo, altanero, usw.

Ein überheblicher Ton ist meistens bei Leuten zu finden, die sich anderen gegenüber als 'höher' einschätzen, und er wirkt daher meistens arrogant und unhöflich. Der überhebliche Ton fußt somit auf der konzeptuellen Metapher HOHER STATUS IST OBEN, NIEDRIGER STATUS IST UNTEN (Lakoff \& Johnson 1980: 1421). So wird die gegensätzliche Bedeutung von hablar en un tono altivo/altanero durch den Phraseologismus bajar el tono (de voz) (dt. den Ton erniedrigen/mäßigen) ausgedrückt und dabei als etwas Positives empfunden.

Bemerkenswert ist die Tatsache, dass Höflichkeit in der Regel prinzipiell kontrollierbar ist und an die Metapher KONTROLLE ODER MACHT AUSÜBEN IST OBEN angepasst sein sollte, jedoch wird das Konzept der 'Höflickheit' im Spanischen mit Unterwürfigkeit in Verbindung gebracht (bajar el tono de voz - 'den Ton absenken'), die nach UNTEN gerichtet ist. Stattdessen benutzt man im Deutschen den Phraseologismus den Ton mäßigen, der auf Kontrolle/Steuerung hindeutet:

(21) CSU-Fraktionschef Georg Schmid nannte den Nazi-Vergleich durch den Kirchensprecher eine „unmögliche Äußerung“. Zwar habe Claudia Roth selbst eine Grenze überschritten, er halte aber beide Äußerungen für keinen „guten Umgang“. Er appellierte an die Beteiligten, den Ton zu mäßigen. (NUN07/OKT.03953 Nürnberger Nachrichten, 24.10.2007, S. 17)

Der Ton kann im Spanischen auch als ein Gefäß konzeptualisiert werden. Der idiomatische Ausdruck salidas de tono (wörtl. übersetzt „Ausgang des Tones") erzeugt eine klare Differenzierung zwischen dem Inneren und dem Äußeren eines 'Gegenstandes' Ton und aktualisiert somit die konzeptuelle Metapher DER TON IST EIN OBJEKT (IN EINEM BEHÄLTER), die aus der Archimetapher EINE AUSSAGE IST EIN GEFÄSS abgeleitet wird. Dem Phraseologismus salidas de tono liegt der Gedanke zugrunde, dass der Ton in dem Moment außer Kontrolle 
gerät, in dem er aus dem Behälter entweicht. Salidas de tono trägt daher die metaphorische Bedeutung 'sich unangemessen/unadequät verhalten':

(22) Las constantes salidas de tono de Schroeder, sus ataques a la línea del partido y la manera descarada en que se postula él mismo como el mejor candidato a canciller, agotaron la paciencia de Scharping. Este anunció el jueves, entre aplausos, que destituía a Schroeder como máximo portavoz del SPD para asuntos económicos. El «señor de Hannover» ha perdido una batalla pero nadie en Bonn se atreve a predecir que la guerra haya terminado. Las bases del SPD están divididas. (CREA La Vanguardia 02.09.95)

Das Konzept BEHÄLTER findet sich ebenso bei dem Phraseologismus fuera de tono. Als Äquivalent zu den Ausdrücken salidas de tono und fuera de tono kann der deutsche Phraseologismus sich im Ton vergreifen dienen. Im Spanischen hat das phraseologische Äquivalent palabras destempladas (dt. 'vergriffene Wörter') eine ähnliche Bedeutung (mit identischem Bild). Zum Bildschema [VERSTIMMT SEIN] gehören im Spanischen die Verben desafinar und destemplar, die auf die metaphorische Bedeutung der Ausdrücke 'die Mäßigung verlieren' oder 'sich unangemessen, indiskret und unpassend äußern' zurückzuführen sind. Im Spanischen steht demgegenüber der idiomatische Ausdruck templar gaitas, der die metaphorische Bedeutung von 'sich beherrschen (bei), im Griff haben (bei), sich mäßigen' besitzt. Das Verb 'templar' trägt die wörtliche Bedeutung von 'ein Instrument stimmen'. Nach Rodríguez-Vidas (2004:213) lässt sich der Ausdruck salida de tono in die systematische Klassifizierung 'palabras destempladas (=raue Töne/Worte)' einpassen.

\section{DER TON IST EIN PHYSISCHES OBJEKT}

Dass Ideen als Objekte konzeptualisiert werden, ist ein häufig zu beobachtendes Phänomen. Wir sprechen beispielsweise von gewichtigen Worten, wenn wir bemerkenswerte Kommentare hervorheben wollen, oder sagen das war jetzt eine hohle Phrase, wenn wir 'inhaltlose Reden' beschreiben wollen.

Im Grunde genommen ist das Konzept IDEEN SIND OBJEKTE auf die ConduitMetapher (Röhrenmetapher) zurückzuführen, welche von Michael Reddy (1979) beschrieben wurde. Die Röhrenmetapher impliziert einen Sprecher, der einerseits die Ideen durch eine Röhre $\mathrm{zu}$ einem Hörer sendet und der andererseits die Ideen/Objekte aus den Worten/dem Gefäß zieht. Reddy stützt sich auf die englische Sprache, in der mindestens 70 Prozent der Ausdrücke aus dem untersuchten Bereich seine Beobachtung belegen. Daraus entwickelte sich ebenso die konzeptuelle Metapher SPRACHLICHE AUSDRÜCKE SIND GEFÄSSE FÜR BEDEUTUNGEN (ich habe eine Idee; wir tauschen unsere Gedanken aus; Diese Idee vermittele ich u.a.).

Als typisches Beispiel für die Metapher IDEEN SIND OBJEKTE (Lakoff \& Johnson 1980: 127) kann der Ausdruck Hast du Töne? im Deutschen betrachtet werden. Der Phraseologismus Hast du Töne? bringt den Aspekt des Besitzes 
durch das Verb haben (,tener') zum Ausdruck. Darüber hinaus wird beim Verstehen dieses Phraseologismus die konzeptuelle Metapher EIN TON IST EIN OBJEKT, DAS MAN IN BESITZ NIMMT aktiviert. Im Spanischen werden an deren Stelle je nach Kontext verschiedene andere Bilder eingesetzt ( No puede ser!, ;Esto es el colmo!, ¡Esto es inaudito!, ¡No me lo puedo creer!, usw.).

Der Phraseologismus Hast du Töne? tritt meistens in solchen Kontexten auf, wo eine Überraschung sowohl positiv als auch negativ bewertet werden kann. Als feststehender Satz beschränkt sich Hast du Töne? auf einen rethorischen Fragesatz, der in der zweiten Person Singular gebildet werden muss.

(23) Haste Töne? Noch über 5 Monate und schon stehen die Kapellen fest! 4 Neulinge und 4 „Wiederholungstäter", genauer gesagt: 4 aus dem Kreisverband Rems-Murr, 2 aus dem Kreisverband Ostalb und 2 aus dem Verband Stuttgart werden aufspielen. (GOOGLE / https://de-de.facebook.com/pluederhaeuser.festtage)

Im Spanischen benutzt man dabei den Ausdruck no tener palabras para algo, um auf die gleiche metaphorische Bedeutung hinzuweisen. Dennoch lassen sich pragmatische Aspekte des Phraseologismus no tener palabras (para algo) feststellen, die sich von dem deutschen Ausdruck unterscheiden. Die spanische Wendung richtet sich nicht auf einen bestimmten Hörer und drückt meistens eine negative Überraschung aus, welche eine unerwartete Handlung oder Situation beschreibt. Ferner wird hier keine rhetorische Frage verwendet. Der Sprecher hat nicht mit der geäußerten Bemerkung oder mit dem Verhalten des Gegenübers oder eines Dritten gerechnet. Und dies führt dazu, dass die Gedanken (Worte/Töne) des Sprechers blockiert werden (sie geraten außer Kontrolle).

(24) Pero no quiere echar más leña al fuego que aún arde con fuerza y prefiere morderse la lengua antes de valorar el cambio de actitud de su compañera Irene Lozano, la diputada nacional que ahora quiere disputar el liderazgo a la actual directiva cuando hace unos meses se indignó públicamente con el eurodiputado Sosa Wagner por pedir abrir conversaciones con Ciudadanos, justo lo mismo que ella defiende ahora. «No tengo palabras para definir a Irene Lozano», zanjó. (El Confidencial 16.04.15)

Darüber hinaus haben wir festgestellt, dass die Töne als Objekte etwas bewirken können. Der idiomatische Ausdruck Der Ton macht die Musik realisiert die Metonymie DER TON STEHT FÜR DIE PERSON. Dem Ausdruck Der Ton macht die Musik liegt darüber hinaus die konzeptuelle Metonymie DIE WIRKUNG STEHT FÜR DIE URSACHE zugrunde (Lakoff and Johnson 1980: 36). Die Quelle der metonymischen Motiviertheit ist der Ton, der durch die Lautstärke der Stimme und durch die Tonlage eine herausragende Rolle auf die verbale Kommunikation besitzt. Diese Redewendung bezieht sich darauf, dass sich der eigentliche Inhalt des Gesagten als zweitrangig erweist, denn das Allerwichtigste besteht darin, den richtigen Tonfall (hierzu zählen die Betonung, die Sprachmelodie und der Sprachrhythmus) und die richtige Haltung $\mathrm{zu}$ finden, wenn man etwas 
Bestimmtes mitteilen will. Im Spanischen kann der Ausdruck No es lo que se dice sino cómo se dice als Entsprechung dienen, in der zwei Aspekte, nämlich 'der Inhalt' und 'die Sprechweise', im Ausdruck vorhanden sind.

Zurecht äußert sich Jessen (2012: 47-48) bezüglich der Wichtigkeit der menschlichen Stimme in der Kommunikation wie folgt:

Der Ton macht die Musik, Stimme erzeugt Stimmung - diese Weisheiten kann niemnad ausser Kraft setzen und es gilt: nur in ruhiger und vertrauensvoller Stimmung werden Menschen das kundtun, was in Ihnen noch unvollständig, unfertig, konfliktbeladen usw. Rumort. Ruhiger, warme Stimme trägt zu guter Stimmung bei; es gibt Stimmen, die das Gefühl vermitteln, man verliert Kraft und es gibt Stimmen, die das Gefühl geben, man baue Kraft auf. Natürliche Rhetorik übt auch diese Wirkung.

Nur vor dem Hintergrund, dass ein Ton einen Klang und eine Lautstärke hat, der menschliche Beziehungen determiniert (Höflichkeit), kann verständlich werden, was das Wort Ton bedeutet, wenn wir uns auf die konzeptuelle Metapher DIE (UN)HÖFLICHKEIT IST EIN MUSIKALISCHER TON beziehen. In ihrer Konzeptualisierung ist der Ton dem Umgangston gleichzusetzen.

Dementsprechend ergibt sich die konzeptuelle Metapher HÖFLICHKEIT IST EINE AKUSTISCHE WAHRNEHMUNG, welche auch in den folgenden Phraseologismen zu finden ist:

Dt. der feine Ton, der gute Ton, zum guten Ton gehören, den richtigen Ton anschlagen, einen höflichen Ton anschlagen, den richtigen Ton finden/treffen, der Ton macht die Musik; Sp. de buen tono, decir/contestar algo con buen tono, emplear el tono adecuado/apropiado/correcto, acertar con el tono, dar (con) el tono adecuado

Sowohl im Deutschen als auch im Spanischen finden sich der Ausdruck den richtigen Ton treffen/finden (,encontrar/dar con el tono adecuado'), in denen die kognitive Metapher DER TON IST EIN OBJEKT realisiert ist, wobei das Verb treffen, worauf sich der Phraseologismus den richtigen Ton treffen stützt, auf der konzeptuellen Metapher ARGUMENTIEREN IST KRIEG basiert.

Das Auftreten von 'anschlagen' im Idiom den richtigen Ton anschlagen (,dar con el tono acertado') macht die konzeptuelle Metapher DER MENSCH IST EIN MUSIKINSTRUMENT bzw. DER MENSCH IST EIN SAITENINSTRUMENT deutlich. Dabei bezieht sich das Verb anschlagen genau genommen sowohl auf Schlaginstrumente als auch um Tasteninstrumente, bei welchen der Ton durch Anschlagen einer oder mehrerer Tasten auf einer Klaviatur oder auf einem Schlaginstrument indirekt erzeugt werden.

In Bezug auf das Konzept TRANSPORT haben wir ebenfalls den Ausdruck den Ton angeben (llevar la voz cantante) festgestellt. Das spanische Äquivalent hat zwar die gleiche metaphorische Bedeutung, ist aber von seinem Bild her unterschiedlich zum Deutschen. Dem deutschen Phraseologismus den Ton angeben steht ein Scheinäquivalent (auch „falscher Freund“ genannt) im 
Spanischen dar la nota gegenüber, denn das Spanische meint die metaphorische Bedeutung 'unangenehm auffallen' oder 'eine Schau abziehen'.

Abschließend lassen sich noch weitere interessante Phraseologismen betrachten: Dt. etw. sorgt für Misstöne, einen Grundton erkennen lassen, zum Grundton gehören, ein Grundton durchzieht etw.; sp. ser la nota discordante/dominante, ser la tónica, usw.

Auf der Grundlage der Musiklehre soll kurz ausgeführt werden, dass eine Tonleiter stufenweise strukturiert ist und dass auf der ersten, vierten und fünften Stufe die Tonika (der Grundton), die Subdominante und die Dominante stehen. In den genannten Ausdrücken sind mindestens zwei konzeptuelle Metaphern enthalten, nämlich DER GRUNDTON IST POSITIV und DER MISSTON IST NEGATIV:

(25) La cordialidad fue la nota dominante de la asamblea, sólo interrumpida en la segunda y última sesión del domingo en dos momentos. (CREA - El País 16.12.1980)

(26) La nota discordante la produjo un grupo de unas cuarenta personas que se proclamaron como no militantes de partidos políticos, quienes una vez finalizada la manifestación, golpearon varios coches y agredieron a los conductores, uno de ellos redactor de EL PAÍS, que fue golpeado en la cabeza. (CREA - El País 10-09-1977)

(27) Juncker gibt damit einen Grundton der Gelassenheit vor, der der CSU durch diesen Parteitag helfen kann. (Merkur Online 12.12.2014)

(28) Es wird erwartet, dass Kerry bereits in Kürze vom Senat bestätigt wird. Das Ende der Amtszeit der Ministerin endet allerdings mit einem Misston: (Die Zeit, 28.01.2013)

\section{SCHLUSSBEMERKUNGEN}

Unsere Beschreibung zahlreicher Phraseologismen, welche das Konzept Ton aus den Bereichen der Sprache und der Musik enthalten, erweist sich als produktives sprachliches Mittel. Es steht außer Zweifel, dass die Musik und die Sprache durch Metapher semantisch motiviert sind. Der Sprachvergleich zeigt, dass im Spanischen und Deutschen überwiegend ähnliche metaphorische Bedeutungen zum Einsatz kommen.

Wir haben untersucht, inwieweit das Konzept Ton als Sprachklang, musikalischer Parameter, oder als Stimme in beiden Sprachen konzeptualisiert werden kann. Die konzeptuellen Strukturen, auf die wir uns bezogen haben —SUBSTANZ, GEFÄSS, VERTIKALE ACHSE (oben-unten), OBJEKT- haben gezeigt, dass dadurch konkrete Aspekte der menschlichen Handlungen, des Charakters oder der Gefühle in den Vordergrund gerückt werden können.

Auf der Ebene der konkreten Metaphorik treten in beiden Sprachen klare Konvergenzen auf, wie die Klangfarbe (harter Ton, tono duro), der Ton (als Stimme), als Träger von Emotionen (warmer Ton, tono cálido), der Ton (als Stimme) als Ausdruck von Verhalten (scharfer Ton; tono agudo, cortante) usw. Es wird dabei evident, dass bestimmte Tastsinneswahrnehmungen in beiden 
Sprachen als positiv (warm, glatt, weich; cálido, blando, suave) oder als negativ (hart, rau, scharf; áspero, grosero, duro, frío) konzeptualisiert werden.

Die Umgangsformen bzw. das Konzept der Höflichkeit spielen eine herausragende Rolle in beiden Sprachen hinsichtlich der vertikalen Achse OBENUNTEN. Das gewöhnliche Ergebnis der metaphorischen Interpretation von hoher Ton basiert auf der Lautstärke, und dies lässt sich wiederum auf die Merkmale UNHÖFLICKEIT, SCHLECHTE MANIEREN, UNANGEBRACHTE SPRECHWEISE zurückführen, da sowohl die Höhe als auch die Lautstärke als Kategorien auf einer vertikalen Achse konzeptualisiert werden können. Arroganz steht ebenso oben wie die Ausdrücke in überheblichem Ton reden, hablar en un tono altivo zeigen.

Es ist deutlich geworden, dass es konzeptuelle Parallelen zwischen den Phraseologismen der einen und der anderen Sprache gibt, und zwar hauptsächlich aufgrund der Tatsache, dass der Musik und der Sprache (Ton, Stimme, Sprachklang) universelle Eigenschaften zugeschrieben werden können.

Anhand zahlreicher Beispiele haben wir einige der zentralen Annahmen, Ergebnisse und Probleme der aktuellen kognitiven Linguistik illustriert, wie z.B. die Interaktion mentaler Repräsentationen, die Bildung mentaler Modelle, sprachliche Interpretationsergebnisse, welche bei der Metaphorik aufeinandertreffen, in dem Sinne, dass die Töne in der Tat kognitiv erfasst werden können: als Substanzen, Objekte oder räumliche Entitäten.

Man kann zum Schluss erwähnen, dass das Potential der Bildhaftigkeit des Konzeptes Ton sehr hoch ist, obgleich wir uns nicht auf der Ebene des Visuellen sondern auf der Ebene des Hörbaren bewegen.

BIBLIOGRAPHIE

BALDAUF, C. (1997), Metapher und Kognition: Grundlagen einer neuen Theorie der Alltagsmetapher, Frankfurt am Main: Peter Lang.

BARCELONA, A. (2003), "On the plausibility of claiming a metonymic motivation for conceptual metaphor", in Barcelona, A. (ed.): Metaphor and Metonymy at the Crossroads: A Cognitive Perspective, Berlin: Mouton de Gruyter, 31-58.

CYTOWIC, R. (2002), Synesthesia: A Union of the Senses, Cambridge: MIT Press.

DAY, S. (1997), "Synaesthesia: Phenomenology and Neuropsychology - A Review of Current Knowledge", in Baron-Cohen, S., Harrison, J. E. (ed.): Synaesthesia. Classic and Contemporary Readings. Oxford, 17-39.

EHRHARDT, C. und NEULAND, E. (2009), Sprachliche Höflichkeit in interkultureller Kommunikation und im DaF-Unterricht. Frankfurt: Peter Lang.

FILLMORE, C. (1982), “Frame semantics, en Linguistic Society of Korea” (ed.): Linguistics in the Morning Calm, Seoul, Hanshin Publishing, 111-137.

JesSEN, F. (2012), Schlüssel-Qualifikation Kommunikation. Professionell kommunizieren mit Mitarbeitern, Berlin: Epubli.

GEGENFURTNER, K. R. (2003), Gehirn und Wahrnehmung, Frankfurt am Main: Fischer Taschenbuch. 
GOLDSTEIN, B. E. (2002), Wahrnehmungspsychologie, Heidelberg: Spektrum Akademischer Verlag.

GROSS, S. (2002), “Literatur und Synästhesie: Überlegungen zum Verhältnis von Wahrnehmung, Sprache und Poetizität", in Adler, H. /Zeuch, U. (ed.): Synästhesie: Interferenz, Transfer, Synthese der Sinne, Würzburg, Königshausen und Neumann, 57-95.

GRUTSCHUS, A. (2009), Strategien der Musikbeschreibung: Eine diachrone Analyse französischer Toneigenschaftsbezeichnungen, Berlin: Frank und Timme.

LAKOFF, G.; JOHNSON, M. (1980), Metaphors We Live By. Chicago, University of Chicago Press.

Mansilla PÉRez, A.; Mellado Blanco, C. (2015), “El campo conceptual Agresión VERBAL en la fraseología alemán-español desde un enfoque cognitivo", en Neuphilologische Mitteilungen, CXVI/I, 113-32.

MÖBIUS, B. (1993), Ein quantitatives Modell der deutschen Intonation: Analyse und Synthese von Grundfrequenzverläufen. Tübingen: Niemeyer.

REDDY, M. J. (1979), “The Conduit Metaphor: A Case of Frame Conflict in Our Language About Language", in Ortony, A. (ed.): Metaphor and Thought, Cambridge: Cambridge University Press, 284-324.

RIELÄNDER, M. M. (1982), Reallexikon der Akustik, Frankfurt am Main: Erwin Bachinsky. RODRÍGUEZ-VIDA, S. (2004), Diccionario temático de frases hechas, Barcelon: Columbus.

SCHEMANN, H.; Mellado Blanco, C.; Buján/P.; IGLESIAS, N.; LARRETA, J. P.; MANSILla, A. (2013), Idiomatik Deutsch-Spanisch, Hamburg: Buske Verlag.

ULLMANN, S. (1964), Language and Style, Oxford: Basil Blackwell.

VIEREGGe, WilHELM H. (2000), "Sprache und Musik: Ein Vergleich im Hinblick auf ihren semiotischen und ästhetischen Charakter", in Pahn, J. /Lamprecht-Dinnessen, A./Keilmann, A./Bielefeld, K./Seifert, E. (ed.): Sprache und Musik. Beiträge der 71. Jahrestagung der Deutschen Gesellschaft für Sprach- und Stimmheilkunde e.V. Berlin, 12.13. März 1999. Stuttgart, Steiner, 163-173.

VoGT, S. (2013), "Die Analyse 'synästhetischer' Metaphern mittels Frames", in Metaphorik.de 23, 19-48.

ZAREND, A. (2015), Höflichkeit in der interkulturellen Kommunikation Russisch - Deutsch, Berlin, Frank und Timme.

ZBIKOWSKI, L. (1997), "Conceptual models and cross-domain mapping: New perspectives on theories of music and hierarchy", in Journal of Music Theory 41, 193225.

ZBIKOWSKI, L. (2000), “Des Herzraums Abschied: Mark Johnson's theory of embodied knowlegde and music theory", in Theory and Practice 22-23, 1-16. 